\title{
Salvage Chemoradiation Therapy for Recurrence After Radical Surgery or Palliative Surgery in Esophageal Cancer Patients: A Prospective, Multicenter Clinical Trial Protocol
}

\section{Xiao Chang}

Department of Radiation Oncology, National Cancer Center/National Clinical Research Center for Cancer/Cancer Hospital, Chinese Academy of Medical Sciences and Peking Union Medical College, Beijing, 100021, China.

\section{Lei Deng}

Department of Radiation Oncology, National Cancer Center/National Clinical Research Center for Cancer/Cancer Hospital, Chinese Academy of Medical Sciences and Peking Union Medical College, Beijing, 100021, China.

\section{Wenjie Ni}

Department of Radiation Oncology, National Cancer Center/National Clinical Research Center for Cancer/Cancer Hospital, Chinese Academy of Medical Sciences and Peking Union Medical College, Beijing, 100021, China.

\section{Chen Li}

Department of Radiation Oncology, National Cancer Center/National Clinical Research Center for Cancer/Cancer Hospital, Chinese Academy of Medical Sciences and Peking Union Medical College, Beijing, 100021, China.

\section{Weiming Han}

Department of Radiation Oncology, National Cancer Center/National Clinical Research Center for Cancer/Cancer Hospital, Chinese Academy of Medical Sciences and Peking Union Medical College, Beijing, 100021, China.

\section{Linrui Gao}

Department of Radiation Oncology, National Cancer Center/National Clinical Research Center for Cancer/Cancer Hospital, Chinese Academy of Medical Sciences and Peking Union Medical College, Beijing, 100021, China.

\section{Shijia Wang}

Department of Radiation Oncology, National Cancer Center/National Clinical Research Center for Cancer/Cancer Hospital, Chinese Academy of Medical Sciences and Peking Union Medical College, Beijing, 100021, China.

\section{Zongmei Zhou}

Department of Radiation Oncology, National Cancer Center/National Clinical Research Center for Cancer/Cancer Hospital, Chinese Academy of Medical Sciences and Peking Union Medical College, Beijing, 100021, China.

\section{Dongfu Chen}

Department of Radiation Oncology, National Cancer Center/National Clinical Research Center for Cancer/Cancer Hospital, Chinese Academy of Medical Sciences and Peking Union Medical College, Beijing, 100021, China.

\section{Qinfu Feng}

Department of Radiation Oncology, National Cancer Center/National Clinical Research Center for Cancer/Cancer Hospital, Chinese Academy of Medical Sciences and Peking Union Medical College, Beijing, 100021, China.

\section{Jun Liang}

Department of Radiation Oncology, National Cancer Center/National Clinical Research Center for Cancer/Cancer Hospital, Chinese Academy of Medical Sciences and Peking Union Medical College, Beijing, 100021, China.

\section{Nan Bi}

Department of Radiation Oncology, National Cancer Center/National Clinical Research Center for Cancer/Cancer Hospital, Chinese Academy of Medical Sciences and Peking Union Medical College, Beijing, 100021, China.

Jima Lv

Department of Radiation Oncology, National Cancer Center/National Clinical Research Center for Cancer/Cancer Hospital, Chinese Academy of Medical Sciences and Peking Union Medical College, Beijing, 100021, China.

\section{Shugeng Gao}

Department of Thoracic Surgery, National Cancer Center/National Clinical Research Center for Cancer/Cancer Hospital,Chinese Academy of Medical Sciences and Peking Union Medical College, Beijing, 100021, China

\section{Yousheng Mao}

Department of Thoracic Surgery, National Cancer Center/National Clinical Research Center for Cancer/Cancer Hospital,Chinese Academy of Medical Sciences and Peking Union Medical College, Beijing, 100021, China

\section{Qi Xue}

Department of Thoracic Surgery, National Cancer Center/National Clinical Research Center for Cancer/Cancer Hospital,Chinese Academy of Medical Sciences and Peking Union Medical College, Beijing, 100021, China

Zefen Xiao ( $\sim$ xiaozefen@sina.com) 
National Cancer Center/National Clinical Research Center for Cancer/Cancer Hospital, Chinese Academy of Medical Sciences and Peking Union Medical College, Beijing, 100021, China https://orcid.org/0000-0003-0503-6814

\section{Study protocol}

Keywords: esophageal neoplasm, locoregional recurrence, R1/R2 resection, chemoradiation therapy, palliative management

Posted Date: June 17th, 2020

DOI: https://doi.org/10.21203/rs.3.rs-35239/v1

License: (c) (i) This work is licensed under a Creative Commons Attribution 4.0 International License. Read Full License

Version of Record: A version of this preprint was published at BMC Cancer on September 14th, 2020. See the published version at https://doi.org/10.1186/s12885-020-07315-0. 


\section{Abstract}

Background: Currently, adjuvant therapy is not recommended for patients with thoracic esophageal squamous cell carcinoma (TESCC) after radical surgery, and a proportion of these patients go on to develop locoregional recurrence (LRR) within 2 years. Besides, there is no evidence for salvage chemoradiation therapy (CRT) in patients with residual tumor after esophagectomy (R1/R2 resection). In addition, factors like different failure patterns and relationship with normal organs influence the decision for salvage strategy. Here, we aimed to design a modularized salvage CRT strategy for patients without a chance of salvage surgery according to different failure patterns (including R1/R2 resection), and further evaluated its efficacy and safety.

Methods: Our study was designed as a one arm, multicenter, prospective clinical trial. All enrolled patients were stratified in a stepwise manner based on the nature of surgery (R0 or R1/2), recurrent lesion diameter, involved regions, and time-to-recurrence, and were further assigned to undergo either elective nodal irradiation or involved field irradiation. Then, radiation technique and dose prescription were modified according to the distance from the recurrent lesion to the thoracic stomach or intestine. Ultimately, four treatment plans were established.

Discussion: This prospective study provided high-level evidence for clinical salvage management in patients with TESCC who developed LRR after radical surgery or those who underwent R1/R2 resection.

Trial registration: ClinicalTrials.gov, NCT03731442, Registered 12 Octomber2018, https://clinicaltrials.gov/NCT03731442

\section{Background}

According to the 2019 National Comprehensive Cancer Network (NCCN) guidelines for esophageal cancer ${ }^{[1]}$, adjuvant treatment is not recommended for patients with thoracic esophageal squamous cell cancer (TESCC) who received radical surgery as their first treatment, regardless of the T and N status. However, the recurrence rate is as high as $23.8-58 \%$, and the median time to recurrence is about 10.5 months ${ }^{[2]-[6]}$. Even in Japan where three-field lymphadenectomy is the preferred treatment option, $24-46 \%$ patients go on to experience recurrence after R0 resection, which is the main cause of surgical treatment failure ${ }^{[7]-[10]}$. Besides, in patients with residual tumor (R1/R2), salvage chemoradiation therapy (CRT) is recommended as the main component of palliative management for locoregional recurrence (LRR) disease. However, data of large samples or high-level evidence are still lacking.

Our previous retrospective analysis ${ }^{[11]}$ indicated that most patients develop lymph node recurrence in the supraclavicular (25.8\%) and upper mediastinal (44.4\%) regions, and those who underwent salvage CRT had significantly better survival than those that underwent radiotherapy alone, chemotherapy, or best supportive care. Similar results were found in other studies ${ }^{[12]-[15]}$. Overall survival (OS) directly depended on failure patterns and corresponding treatment strategies, so prospective clinical trials were necessary for screening of specific patients to attain survival benefit from the optimal salvage strategy.

This study was aimed to design a modularized salvage CRT strategy for patients unsuited for salvage surgery based on different failure patterns (including R1/R2 resection) and further evaluate its efficacy and safety.

\section{Methods/design}

\section{Study design and objectives}

This study was designed as a one-arm, multicenter, prospective clinical trial. The enrolled patients were stratified in a stepwise manner based on the nature of surgery (R0 or R1/2), recurrent lesion diameter, involved regions, and time-to-recurrence, and were further assigned to undergo either elective nodal irradiation (ENI) or involved field irradiation (IFI). Then, radiation technique and dose prescription were modified according to the distance from the recurrent lesion to thoracic stomach or intestine. Ultimately, four treatment plans were established. A flow chart of the overview of the study design is shown in Figure 1.

The primary objective of this trial is to observe the 1-, 2-, and 3-year OS. The secondary objectives include the 1-, 2-, and 3-year rates of progression-free survival (PFS), completion rates, out-field recurrence, and toxicity profiles.

The study began on November 2018, and patients will continue to be included until November 2022.

\section{Patient Population}

Patients enrolled thus far mainly comprise untreated patients after LRR or palliative surgery. The inclusion criteria include: (1) R1/R2 resection, (2) LRR after radical surgery, (3) out-field LRR after adjuvant chemoradiation or radiotherapy, (4) LRR after adjuvant chemotherapy, (5) no prior therapy after LRR, (6) age 16-70 years, (7) good general condition (i.e., Karnofsky Performance Status [KPS] $\geq 70)$ ], (8) normal complete blood count (CBC), especially white blood cell count $>4.0^{*} 10^{\wedge} 9 / \mathrm{L},(9)$ satisfactory liver and kidney functions.

The exclusion criteria include: (1) prior malignancies within 5 years, (2) pregnant status or lactation, (3) history of drug allergy, (4) refused informed consent (5) distant lymph node (other than metastasis to supraclavicular or celiac lymph nodes) or visceral metastasis (including metastasis to bone, lung, liver, pleura, or peritoneum) (6) severe cardiovascular diseases, infections, active ulcerations, diabetes mellitus with unstable blood sugar, and mental disorders.

\section{Recurrence}

Tumor residue includes positive pathological margins of the specimens (R1) and incomplete tumor resection during the operation (R2). LRR is defined as recurrence at sites of the anastomosis, tumor bed, mediastinal lymph nodes, or para-gastric lymph nodes (including nodes adjacent to the cardia or along the course of the left gastric artery). Recurrence in the deep cervical, supraclavicular, or celiac regions are also defined as regional relapse. Distant metastasis was 
defined as metastasis in the liver, lung, bone, and pleura; subcutaneous metastasis; and other nonregional lymph node metastasis such as axillary and inguinal lymph nodes. If a second recurrence was detected within 4 weeks after the first occurrence, it was considered synchronous. Once suspicious recurrent lesions are identified on imaging, biopsy is attempted. The diagnostic standard for imaging should meet the criteria of significant enlargement or increase in the number of existing lymph nodes, or the appearance of the new lymph nodes compared with previous examinations. Otherwise, positron emission tomography-computed tomography (PET-CT) clearly diagnoses recurrence through metabolic activity and imaging features.

To comprehensively describe the design of target volume, the $8^{\text {th }}$ American Joint Committee on Cancer (AJCC) regional lymph node stations ${ }^{[16]}$ were reclassified into four regions (Fig. 2). Region I includes the area above the sternal notch, including the supraclavicular space and No. 1 lymphatic drainage region; region II includes the mediastinal No. 2, 4, and $8 \mathrm{U}$ lymphatic drainage regions; region III includes mediastinal No. 7, 8 M/Lo, and 9 lymphatic drainage regions; and region IV includes the abdominal No. 15-20 lymphatic drainage regions. Close region recurrence was defined as recurrences within the sites of (1) regions I and II, (2) regions II and III, (3) regions III and IV, or (4) regions I and III. Distant regional metastasis was defined as recurrences at the both sites of regions I and IV or region II and IV.

\section{Radiotherapy}

Patients were placed in the supine position with their arms straight beside the body during planning CT, which was recommended to be fused with planning magnetic resonance imaging (MRI) or PET-CT, if available, to further improve the contouring accuracy. The gross tumor volume (GTV-T) or metastatic regional nodes (GTV-N) is defined as the residual tumor, tumor-bed recurrence, or metastatic lymph node. The planning gross tumor volume (PGTV) is created by expanding GTV-T/GTV-N by a uniform 0.5 -cm margin. As for delineation of clinical target volume (CTV), both IFI and ENI were adopted.

In the ENI group, the principle to design prophylactic target volume of high-risk lymphatic drainage regions basically comprised GTV-T/GTV-N plus a 3.0-5.0$\mathrm{cm}$ craniocaudal and $0.6-\mathrm{cm}$ horizontal margin. For recurrence in regions I or II, CTV comprised the region with the upper boundary in the upper margin of the T1 vertebral body or $1.0-1.5-\mathrm{cm}$ superior to GTV-N and lower boundary in the $2.0-3.0-\mathrm{cm}$ inferior to the carina, including the supraclavicular space and No. 1 , $2,4,7$, and $8 \mathrm{U}$ stations. For recurrence in region III, the CTV comprised the region with upper boundary at the level of the clavicular head and lower boundary in the margin 2.0-cm inferior to the carina or 1.0-1.5-cm inferior to GTV-N, including No. 2, 4, 7, and 8U/M stations. For recurrence in region IV, CTV comprised the region with upper boundary in the 1.0-1.5-cm superior to GTV-N and lower boundary in the celiac axis or 1.5-cm inferior to GTV-N, including No. 15-20 stations. The technique of intensity-modulated radiation therapy (IMRT) with simultaneously integrated boost (SIB) or sequential boost was modified according to the safety of the thoracic stomach or intestine. Figure 3A shows the SIB-IMRT being applied to a recurrent lesion far from the thoracic stomach with a prescription dose of PTV 50.4 Gy/1.8 Gy/28 $\mathrm{f}$ and PGTV 59.92-62.16 Gy/2.14-2.22 Gy/28 f. Figure 3B shows the IMRT with sequential boost applied to a recurrent lesion close to the thoracic stomach with a prescription dose of PTV 50.4 Gy/1.8 Gy/28 $\mathrm{f}$ and a sequential boost to PGTV 10-12 Gy/1.8-2 Gy/5-7f.

In the IFI group, CTV only consisted of GTV-T/GTV-N plus a $0.6-0.8-\mathrm{cm}$ horizontal margin and 1.0-1.5-cm craniocaudal margin. No prophylactic irradiation was given to the lymph node drainage regions. For lesions located away from the thoracic stomach (Fig. $4 \mathrm{~A}$ ), the prescribed dose was $60 \mathrm{~Gy} / 2 \mathrm{~Gy} / 30 \mathrm{f}$, and for lesions close to the thoracic stomach (Fig. 4B), the prescribed dose was 59.4-61.2 Gy/1.8 Gy/33-34 f. Planned chest CT at $50 \mathrm{~Gy}$ followed by the radiation field should be modified according to the tumor response. The PTV is derived from CTV plus a uniform 0.5-cm margin.

Organs at risk (OAR) including the lungs, heart, spinal cord, and reconstructed thoracic stomach should be contoured on planning CT. The volume of lung tissue receiving $\geq 20$ Gy should not exceed $28 \%$ of the total lung volume (V20<28\%). The mean dose of lung tissue should be lower than $17 \mathrm{~Gy}$ (Dmean lung $<17 \mathrm{~Gy}$ ). Other dose constraints to OAR will be: V40 heart $<30 \%$, V30 heart $<40 \%$, Dmax spinal cord PRV $<45$ Gy, and V 40 thoracic stomach $<40 \%$ without hot point. If the $\mathrm{V} 50$ thoracic stomach $>50 \%$, the total dose should be reduced up to $45 \mathrm{~Gy} / 1.8 \mathrm{~Gy} / 25 \mathrm{f}$.

\section{Chemotherapy}

Concurrent with radiotherapy, patients will receive two cycles of paclitaxel (135-150 mg/m², d1) and lobaplatin (30 mg/m², d1-2, total dose should not exceed $50 \mathrm{mg}$ ) or nedaplatin $\left(50 \mathrm{mg} / \mathrm{m}^{2}, \mathrm{~d} 1-2\right)$ every 3 weeks. Injection with polyethylene glycol recombinant human granulocyte colony-stimulating factor (PEG-rhG$\mathrm{CSF}$ ) for prophylaxis will be recommended $48 \mathrm{~h}$ after chemotherapy. Two or four cycles of consolidated chemotherapy are recommended within 3 months for patients meeting the following criteria:(1) KPS $\geq 70$, (2) able to swallow more than semisolid food or with nasal feeding, and (3) weight loss $\leq 5 \%$ in $1-2$ months after radiotherapy. The regimen is the same as that of the concurrent phase. However, if patients have previously received taxane- and platinum-based chemotherapy regimens and go on to develop LRR within 6 months, the second-line regimen of chemoradiotherapy is recommended.

\section{Toxicity and adverse events}

All treatment-related toxicities and adverse events should be graded according to the toxicity criteria of the Radiation Therapy Oncology Group (RTOG) and Common Terminology Criteria of Adverse Events (CTCAE) version 4.0 and recorded in detail on patients' case report form (CRF). Serious adverse events (SAE) should be reported to the institutional ethical review committee within $24 \mathrm{~h}$ and dealt with in an appropriate manner.

The second cycle of chemotherapy should be adjusted based on the most severe chemotherapy-related toxicity after the first cycle, and it should only be administered when the toxicity subsides to baseline or grade $0 / 1$. The regimen should be administered at $80 \%$ of the original dose in case of grade 3 hematogenous toxicity, and chemotherapy should be terminated in case of grade 4 hematogenous toxicity, grade 2 hepatic or renal dysfunction, and other non-homological grade 3 toxicities. If grade 3/4 radiation pneumonitis develops, both radiotherapy and concurrent chemotherapy should be terminated.

\section{Follow-up}


Tumor regression should be evaluated according to the Response Evaluation Criteria in Solid Tumors (RECIST) Version 1.1 within 1-2 months, and response to treatment should be documented. All patients should be followed-up for at least 5 years after completion of the protocol; the time interval should be every 3 months for the first 2 years, every 6 months for 3-5 years, and once a year thereafter.

Every follow-up should include the following: (i) History-taking including symptoms of cough, fever, hoarseness, dysphagia, or chest tightness. (ii) CBC and comprehensive blood chemistry profile. (iii) Contrast-enhanced CT of the neck, thorax, and abdomen; ultrasound of the neck and abdomen; upper gastrointestinal contrast; bone scan (in case of bone pain or abnormally elevated alkaline phosphatase); and CT or MRI of the brain (in case of any symptoms related to the central nervous system). (iv) Documentation of patients' status of survival, disease progression, subsequent treatment, nutrition, life quality, and late toxic effects.

\section{Statistical analysis \& sample size considerations}

A chi-square test was used to compare categorical data, with or without correction for continuity. Salvage OS was defined as the date of receiving salvage CRT to the date of death or the last clinical follow-up. DFS was measured from the date of receiving salvage CRT to the date of progression or death from any cause or last clinical follow-up. Cumulative survival rates were estimated using the Kaplan-Meier survival curve and compared using the log-rank test.

This trial is designed as a one-arm prospective study, using PASS 11.0.7 to estimate the sample size. We assume that the estimated 1-year OS of the ENI arm and IFI arm will be $70 \%$ and $60 \%$, respectively. Assuming a two-sided significance level of 0.05 and a power of 0.80 , the sample size of the ENI group and IFI group are estimated to be 83 and 199, respectively. Therefore, a total of 282-300 patients would be needed considering patient loss in each group.

\section{Ethics}

The study protocol has been approved by the ethics committee of the Chinese Academy of Medical Sciences (18-175/1753). Written informed consent will be obtained from each patient before enrollment. The study has been registered in ClinicalTrails.gov (NCT03731442).

\section{Discussion}

Although the 2016-2019 NCCN guidelines recommended CRT as the optimal treatment for patients who developed LRR with prior esophagectomy or received R1/R2 resection, the principle of target volume delineation or dose prescription for salvage CRT are still unclear. In addition, radiation oncologists may find it difficult to screen patients with potential curative possibilities according to failure patterns and recurrence time to attain better patient survival. Therefore, more prospective clinical trials are necessary.

$\mathrm{Ni}$ et al. ${ }^{[11]}$ reported that postoperative pTNM stage and salvage treatment regimen were independent prognostic factors for LRR in esophageal cancer after surgery. Besides, close follow-up after surgery for early detection and timely treatment were also crucial factors. However, the optimal radiation dose for recurrent esophageal cancer has not yet been determined. Nemoto et al. ${ }^{[17]}$ reported that the median survival period of patients who received a radiation dose of $\leq 60$ Gy was 2 months longer than those who received $>60 \mathrm{~Gy}$, although this difference was not statistically significant. However, Zhang et al. ${ }^{[18]}$ reported that TESCC patients with LRR showed better OS and PFS in the group of $\geq 60$ Gy than those in the group of $<60 \mathrm{~Gy}$, and Shioyama et al. ${ }^{[19]}$ also demonstrated that the high-dose group (>50 Gy) was associated with better survival. Our previous data ${ }^{[11]}$ showed that patients receiving $>60 \mathrm{~Gy}$ irradiation dose had a significantly greater 5 -year OS than those who received $\leq 60 \mathrm{~Gy}(25.3 \%$ vs. $13.8 \%, \mathrm{P}=0.026)$. Three-dimensional conformal radiotherapy or IMRT could significantly reduce these toxicities because of better dose distribution between the tumor and normal tissue, ${ }^{[20]-[23]}$ and the SIB technique allowed an escalation dose specifically toward the tumor without over irradiating the OARs ${ }^{[23],[24]}$. Our prospective phase I/II trial[25] supported the safety and efficacy of the dose patterns adopted in this trial (95\% PGTV/PTV $59.92 \mathrm{~Gy} / 50.40 \mathrm{~Gy} / 28 \mathrm{f}, \mathrm{EQD} 2=60.62 \mathrm{~Gy}$ ). In addition, for patients who are intolerant to SIB-IMRT, concurrent chemoradiotherapy with a sequential boost of about $10 \mathrm{~Gy}$ was adopted. Welsh et al. ${ }^{[24]}$ reported that $50 \%$ patients experienced local failure and $90 \%$ LRR cases were within GTV after definitive CRT with a prescription dose of $50.4 \mathrm{~Gy}$. This result indicated that the local control rate was unsatisfactory and therapeutic intensification should be carried out for the primary tumor. Therefore, in order to keep the toxicity level stable, we speculated whether it was possible to improve the local control rate and prolong survival by appropriately increasing the radiotherapy dose.

Although CRT was preferred, the role of chemotherapy in palliative management remains controversial. Nemoto et al. ${ }^{[17]}$ reported that combined chemotherapy was correlated with a better 2-year local control rate, but failed to show better survival. However, previously noted trial RTOG $8501^{[26],[27]}$ showed that the 5 -year OS of definitive radiotherapy with or without chemotherapy was $26 \%$ and $0 \%(P<0.001)$, respectively. Our findings appear consistent with other studies $^{[11]-[15]}$ and have indicated that CRT correlates with better survival than radiotherapy alone and is well tolerated in patients who developed LRR. Further, it was also unclear whether patients should receive consolidation chemotherapy. A propensity score-matched analysis ${ }^{[28]}$ showed that consolidation chemotherapy did not further prolong PFS and OS following definitive CRT, and no prospective randomized clinical trials supported the addition of consolidation chemotherapy following salvage CRT. However, there was still high risk of LRR with synchronous distant metastases ${ }^{[3],[7]-[10],[29]}$, so consolidation chemotherapy was only recommended to patients who has a good general status and responded well to the primary treatment.

However, concerning the target volumes of CRT for esophageal cancer, there is no global consensus regarding whether ENI or IFI should be performed ${ }^{[30]-[35]}$. In this trial, target volumes were determined by the goal of treatment. For LRR patients with potential curable possibility, prophylactic irradiation to high-risk lymph node regions should be considered because of the following reasons: (1) The median time to recurrence is short, and most studies reported $50 \%$ patients develop recurrence within 7-12 months. The median time to recurrence in our hospital was even shorter (7 months), and we rechecked cases to find that that a major proportion of patients with LRR were identified by clinical examinations and close follow-up without any symptoms such as dysphagia, obstruction, or pain. (2) The lymphatic metastasis of esophageal cancer occurred early, and lymph node dissection is known to be difficult given the complex 
anatomy of the upper mediastinum. (3) The recurrence rate in multiple lymphatic regions was high. Ni et al. ${ }^{[11]}$ reported that $>50 \%$ patients had recurrence in multiple regions of the upper mediastinum. For patients with widespread recurrence or giant tumor bulk, IFI was mainly applied to relieve symptoms, achieve high completion rate, and thereby prolong survival.

\section{Abbreviations}

TESCC: thoracic esophageal squamous cell carcinoma; TESCC: thoracic esophageal squamous cell cancer; LRR: locoregional recurrence; CRT: chemoradiation therapy; ENI: elective nodal irradiation; IFI: involved field irradiation; NCCN: national comprehensive cancer network; OS: overall survival; SIB: simultaneously integrated boost; IMRT: intensity-modulated radiation therapy; KPS: Karnofsky Performance Status; CBC: normal complete blood count; PETCT: Positron Emission Tomography-Computed Tomography; MRI: magnetic resonance imaging; AJCC: American Joint Committee on Cancer; GTV-T: Gross Tumor Volume; GTV-N: metastatic regional nodes; PGTV: Planning Gross Tumor Volume; CTV: Clinical Target Volume; PTV: Planning Target Volume; OAR: organ at risk; PEG-rhG-CSF: polyethylene glycol recombinant human granulocyte colony-stimulating factor; RTOG: Radiation Therapy Oncology Group; CTCAE: Common Terminology Criteria of Adverse Events; CRF: case report form; SAE: Serious Adverse Events; RECIST: Evaluation Criteria in Solid Tumors;

\section{Declarations}

\section{Ethics approval and consent to participate}

The study protocol has been approved by the ethics committee of the Chinese Academy of Medical Sciences (18-175/1753). Written informed consent has been obtained from all participants enrolled thus far.

\section{Consent for publication}

Not applicable

\section{Availability of data and materials}

Not applicable - data collection is still ongoing

\section{Competing interests}

The authors declare that they have no competing interests.

\section{Funding}

This work was supported by the Capital Fund for Health Improvement and Research [grant number 2016-2-4021]. The manuscript has been peer reviewed by the funding body.

The funding source has no role in study design, data collection, analysis, interpretation, the writing of the manuscript, or the decision to submit the current study.

\section{Acknowledgements}

We thank all the patients who participated in this trial, all participating branch-centers and investigators who devote their time and passion in the implementation of this study.

\section{Authors' contributions}

ZFX made substantial contributions to the conception and design of the study, revised the article critically for important intellectual content, and approved the final version to be published; $X C$ drafted the manuscript; $L D$ participated in designing and conducting the study; $X C$ and $L D$ collected research data. WNJ, $C L$, WMH, LRG, SJW, ZMZ, DFC, QFF, JL, NB, JML, SGG, YSM and QX provided patients; all authors read and approved the final manuscript.

\section{Trial Status}

The study protocol was approved by the institutional review board in October 2018. Recruitment started in November 2018 and is currently ongoing.

\section{References}

1. National Comprehensive Cancer Network: NCCN Clinical Practice Guidelines in Oncology: Esophageal and Esophagogastric Junction Cancers, Version 2, 2018.

2. Hsu PK, Wang BY, Huang CS, Wu YC, Hsu WH. Prognostic Factors for Post-recurrence Survival in Esophageal Squamous Cell Carcinoma Patients with Recurrence after Resection. J Gastrointest Surg. 2011;15(4):558-565. http://www.ncbi.nlm.nih.gov/pubmed/21327531.

3. Miyata H, Yamasaki M, Kurokawa Y, et al. Survival factors in patients with recurrence after curative resection of esophageal squamous cell carcinomas. Ann Surg Oncol. 2011;18(12):3353-3361.

4. Lu J, Tao H, Song D, Chen C. 549-555 Recurrence risk model for esophageal cancer after radical surgery. Chinese J Cancer Res. 2013;25(5):549-555. 
5. Oppedijk V, Van Der Gaast A, Van Lanschot JJB, et al. Patterns of recurrence after surgery alone versus preoperative chemoradiotherapy and surgery in the CROSS trials. J Clin Oncol. 2014;32(5):385-391.

6. Liu Q, Cai XW, Wu B, Zhu ZF, Chen HQ, Fu XL. Patterns of failure after radical surgery among patients with thoracic esophageal squamous cell carcinoma: Implications for the clinical target volume design of postoperative radiotherapy. PLoS One. 2014;9(5):e97225.

7. Bhansali MS, Fujita H, Kakegawa T, et al. Pattern of recurrence after extended radical esophagectomy with three- field lymph node dissection for squamous cell carcinoma in the thoracic esophagus. World J Surg. 1997;21(3):275-281.

8. Nakagawa S, Kanda T, Kosugi SI, Ohashi M, Suzuki T, Hatakeyama K. Recurrence pattern of squamous cell carcinoma of the thoracic esophagus after extended radical esophagectomy with three-field lymphadenectomy. J Am Coll Surg. 2004;198(2):205-211.

9. Baba M, Aikou T, Yoshinaka H, et al. Long-term results of subtotal esophagectomy with three-field lymphadenectomy for carcinoma of the thoracic esophagus. Ann Surg. 1994;219(3):310-316.

10. Kyriazanos ID, Tachibana M, Shibakita M, et al. Pattern of recurrence after extended esophagectomy for squamous cell carcinoma of the esophagus. Hepatogastroenterology. 2003;50(49):115-120.

11. Ni W, Yang J, Deng W, et al. Patterns of recurrence after surgery and efficacy of salvage therapy after recurrence in patients with thoracic esophageal squamous cell carcinoma. BMC Cancer. 2020;20(1):144.

12. Raoul JL, Le Prise E, Meunier B, et al. Combined radiochemotherapy for postoperative recurrence of oesophageal cancer. Gut. 1995;37(2):174-176.

13. Su X-D, Zhang D-K, Zhang X, Lin P, Long H, Rong T-H. Prognostic factors in patients with recurrence after complete resection of esophageal squamous cell carcinoma. J Thorac Dis. 2014;6(7):949-957.

14. Lu J-C, Kong C, Tao H. Radiotherapy with or without concurrent chemotherapy for lymph node recurrence after radical surgery of thoracic esophageal squamous cell carcinoma. Int J Radiat Oncol Biol Phys. 2010;78(3):710-714.

15. Zhang W-W, Zhu Y-J, Yang H, et al. Concurrent radiotherapy and weekly chemotherapy of 5-fluorouracil and platinum agents for postoperative locoregional recurrence of oesophageal squamous cell carcinoma. Sci Rep. 2015;5:8071.

16. Rice TW, Ishwaran H, Ferguson MK, Blackstone EH, Goldstraw P. Cancer of the Esophagus and Esophagogastric Junction: An Eighth Edition Staging Primer. J Thorac Oncol. 2017;12(1):36-42. http://dx.doi.org/10.1016/j.jtho.2016.10.016.

17. Nemoto K, Ariga H, Kakuto Y, et al. Radiation therapy for loco-regionally recurrent esophageal cancer after surgery. Radiother Oncol. 2001;61(2):165-168.

18. Zhang J, Peng F, Li N, et al. Salvage concurrent radio-chemotherapy for post-operative local recurrence of squamous-cell esophageal cancer. Radiat Oncol. 2012;7:93.

19. Shioyama Y, Nakamura K, Ohga S, et al. Radiation therapy for recurrent esophageal cancer after surgery: clinical results and prognostic factors. Jpn J Clin Oncol. 2007;37(12):918-923.

20. Zhang W, Liu X, Xiao Z, et al. Postoperative intensity-modulated radiotherapy improved survival in lymph node-positive or stage III thoracic esophageal squamous cell carcinoma. Oncol Res Treat. 2015;38(3):97-102. https://www.karger.com/DOI/10.1159/000375391.

21. Sher DJ, Koshy M, Liptay MJ, Fidler MJ. Influence of conformal radiotherapy technique on survival after chemoradiotherapy for patients with stage III non-small cell lung cancer in the National Cancer Data Base. Cancer. 2014;120(13):2060-2068.

22. Lai S-Z, Li W-F, Chen L, et al. How does intensity-modulated radiotherapy versus conventional two-dimensional radiotherapy influence the treatment results in nasopharyngeal carcinoma patients? Int J Radiat Oncol Biol Phys. 2011;80(3):661-668.

23. Welsh JW, Riley B, Palmer MB, et al. Intensity Modulated Proton Therapy Allows Dose Escalation and Normal-Tissue Sparing in Locally Advanced Distal Esophageal Tumors. Int J Radiat Oncol. 2010;78(3, Supplement):S808. http://www.sciencedirect.com/science/article/pii/S0360301610028464.

24. Zhang W-Z, Chen J-Z, Li D-R, et al. Simultaneous modulated accelerated radiation therapy for esophageal cancer: a feasibility study. World J Gastroenterol. 2014;20(38):13973-13980.

25. Li C, Deng W, Wang X, et al. A Phase 1/2 Radiation Dose Escalation Trial Using SIB-IMRT Technique With Concurrent Chemotherapy in Unresectable Esophageal Carcinoma. Int J Radiat Oncol. 2017;99(2, Supplement):E166. http://www.sciencedirect.com/science/article/pii/S0360301617320503.

26. Herskovic A, Martz K, al-Sarraf M, et al. Combined Chemotherapy and Radiotherapy Compared with Radiotherapy Alone in Patients with Cancer of the Esophagus. N Engl J Med. 1992;326(24):1593-1598.

http://www.nejm.org/doi/full/10.1056/NEJM199206113262403\%5Cnhttp://www.nejm.org.proxy.library.vcu.edu/doi/full/10.1056/NEJM1992061132624(

27. Cooper JS, Guo MD, Herskovic A, et al. Chemoradiotherapy of locally advanced esophageal cancer: long-term follow-up of a prospective randomized trial (RTOG 85-01). Radiation Therapy Oncology Group. JAMA. 1999;281(17):1623-1627.

28. Chen Y, Guo L, Cheng X, et al. With or without consolidation chemotherapy using cisplatin/5-FU after concurrent chemoradiotherapy in stage II-III squamous cell carcinoma of the esophagus: A propensity score-matched analysis. Radiother Oncol. 2018;129(1):154-160.

29. Oppedijk V, Van Der Gaast A, Van Lanschot JJB, et al. Patterns of recurrence after surgery alone versus preoperative chemoradiotherapy and surgery in the CROSS trials. J Clin Oncol. 2014;32(5):385-391.

30. Yamashita H, Takenaka R, Omori M, et al. Involved-field radiotherapy (IFRT) versus elective nodal irradiation (ENI) in combination with concurrent chemotherapy for 239 esophageal cancers: A single institutional retrospective study. Radiat Oncol. 2015;10(1):1-10. http://dx.doi.org/10.1186/s13014015-0482-9.

31. Onozawa M, Nihei K, Ishikura S, et al. Elective nodal irradiation (ENI) in definitive chemoradiotherapy (CRT) for squamous cell carcinoma of the thoracic esophagus. Radiother Oncol. 2009;92(2):266-269. http://dx.doi.org/10.1016/j.radonc.2008.09.025.

32. Li M, Zhang Y, Zhu H, et al. Feasibility of Elective Nodal Irradiation (ENI) and Involved Field Irradiation (IFI) in Radiotherapy for the Elderly Patients (Aged $\geq$ 70 Years) with Esophageal Squamous Cell Cancer: A Retrospective Analysis from a Single Institute. PLoS One. 2015;10(12):e0143007.

Page 7/10 
http://dx.doi.org/10.1371/journal.pone.0143007.

33. Ji K, Zhao L, Yang C, Meng M, Wang P. Three-dimensional conformal radiation for esophageal squamous cell carcinoma with involved-field irradiation may deliver considerable doses of incidental nodal irradiation. Radiat Oncol. 2012;7(1):1-8.

34. Huang W, Huang Y, Sun J, et al. Atlas of the thoracic lymph nodal delineation and recommendations for lymph nodal CTV of esophageal squamous cell cancer in radiation therapy from China. Radiother Oncol. 2015;116(1):100-106. http://dx.doi.org/10.1016/j.radonc.2015.06.024.

35. Yamashita H, Okuma K, Wakui R, Kobayashi-Shibata S, Ohtomo K, Nakagawa K. Details of recurrence sites after elective nodal irradiation (ENI) using 3Dconformal radiotherapy (3D-CRT) combined with chemotherapy for thoracic esophageal squamous cell carcinoma - A retrospective analysis. Radiother Oncol. 2011;98(2):255-260. http://dx.doi.org/10.1016/j.radonc.2010.10.021.

\section{Figures}

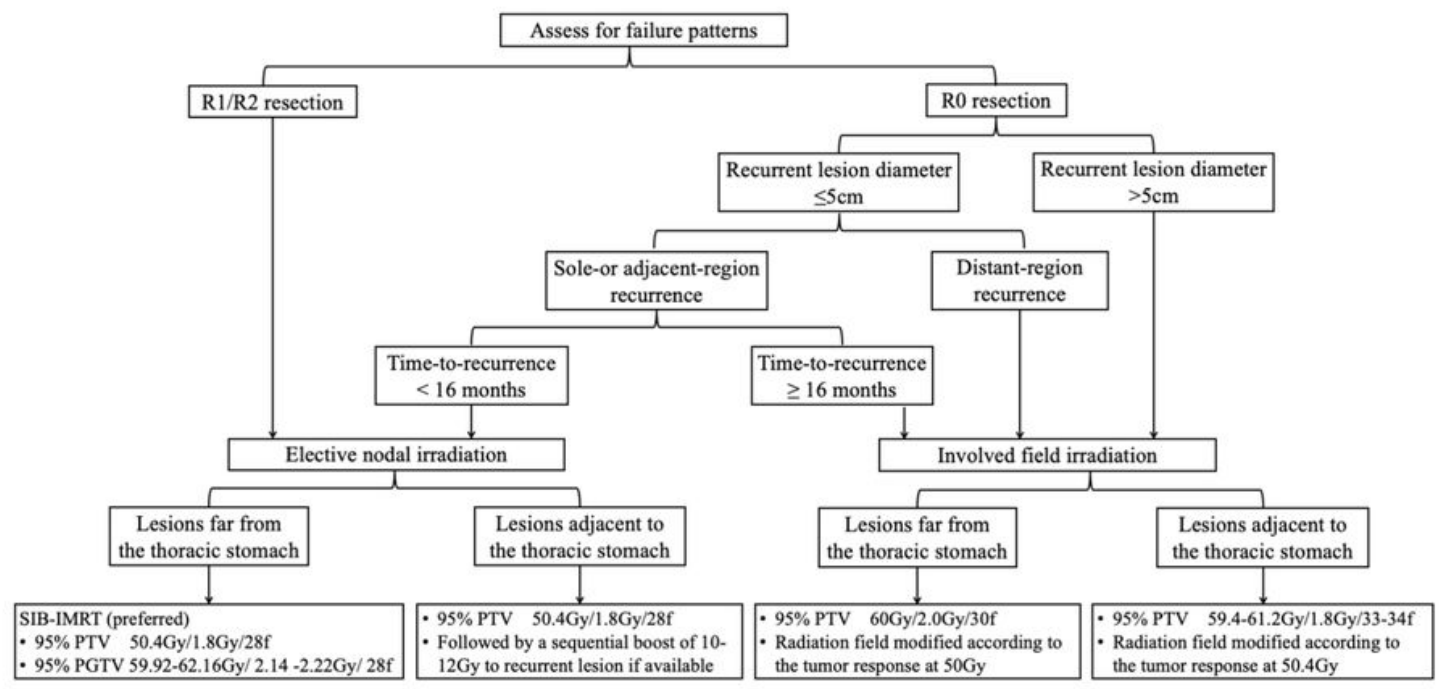

\section{Figure 1}

Flow chart of the trial 


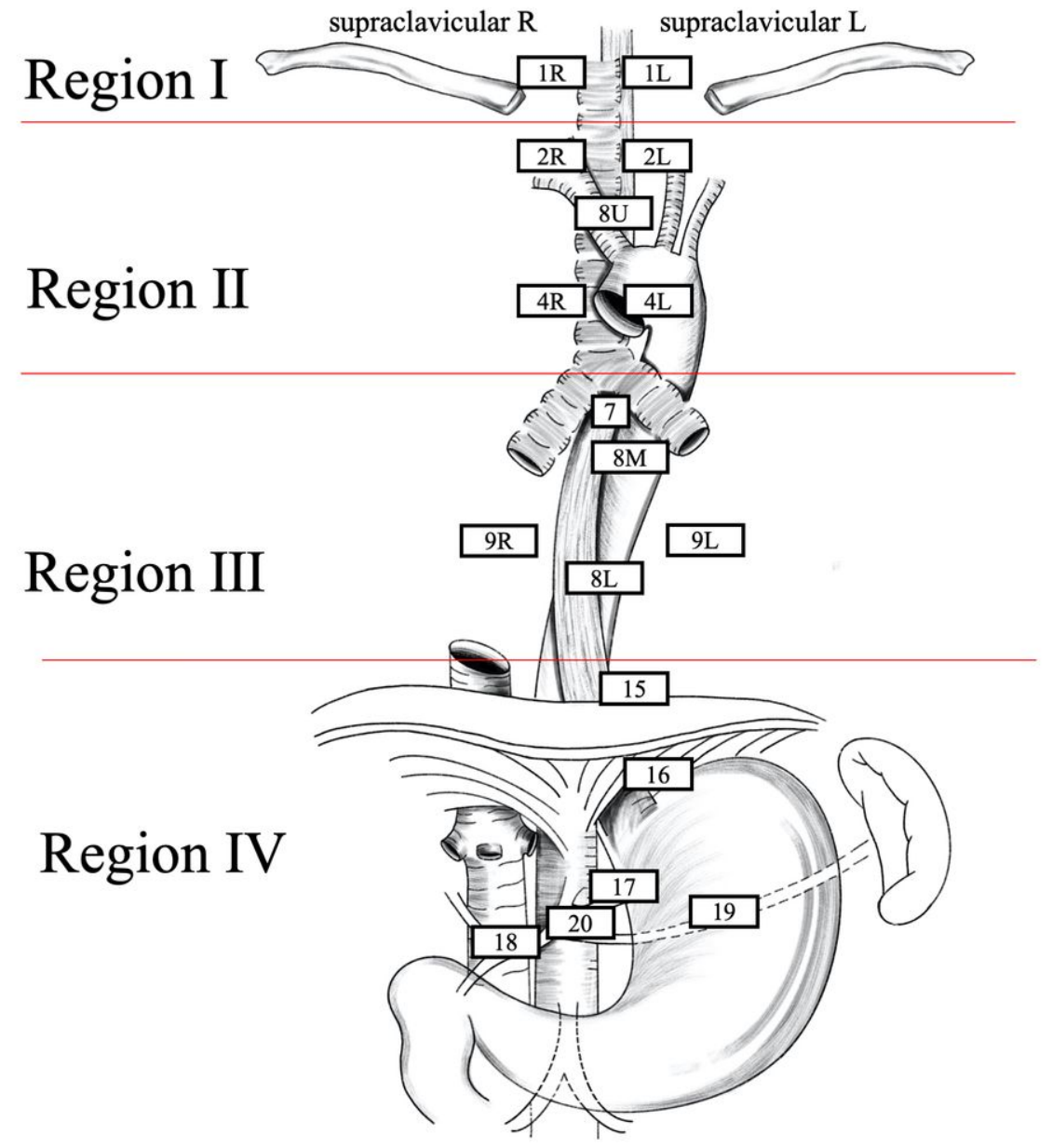

Figure 2

Illustration depicting reclassified regions.

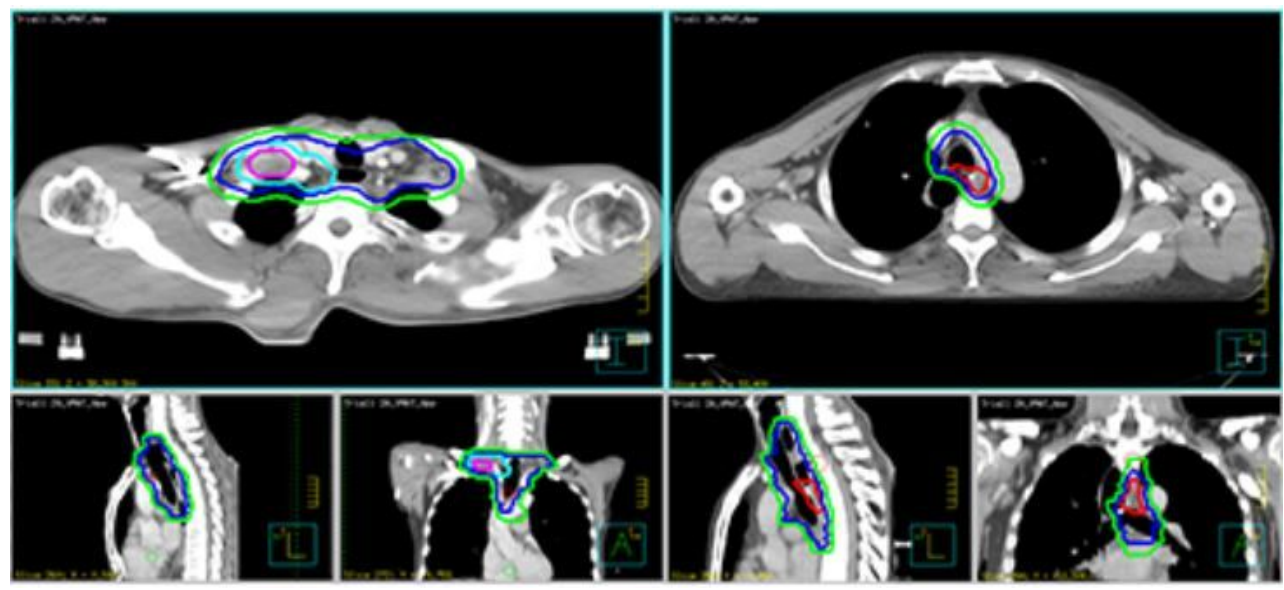

Figure 3

Examples of target volumes delineated in elective nodal irradiation (ENI) pattern are shown based on the planning computed tomography (CT) scans. (A) Lesions far from the thoracic stomach recurred in right supraclavicular space. The ENI field includes bilateral supraclavicular space, $1 R / L, 2 R / L, 4 R / L$, partial 7, and $8 \mathrm{U} / \mathrm{M}$. (B) Lesions adjacent to the thoracic stomach recurred in Station $8 \mathrm{U}$, ENI field includes bilateral supraclavicular space, 1R/L, 2R/L, 4R/L, partial 7, and 8U/M. Pink outlines GTV-N, Red outlines GTV-T, blue outlines CTV, sky blue outlines PGTV, and green outlines PTV. 


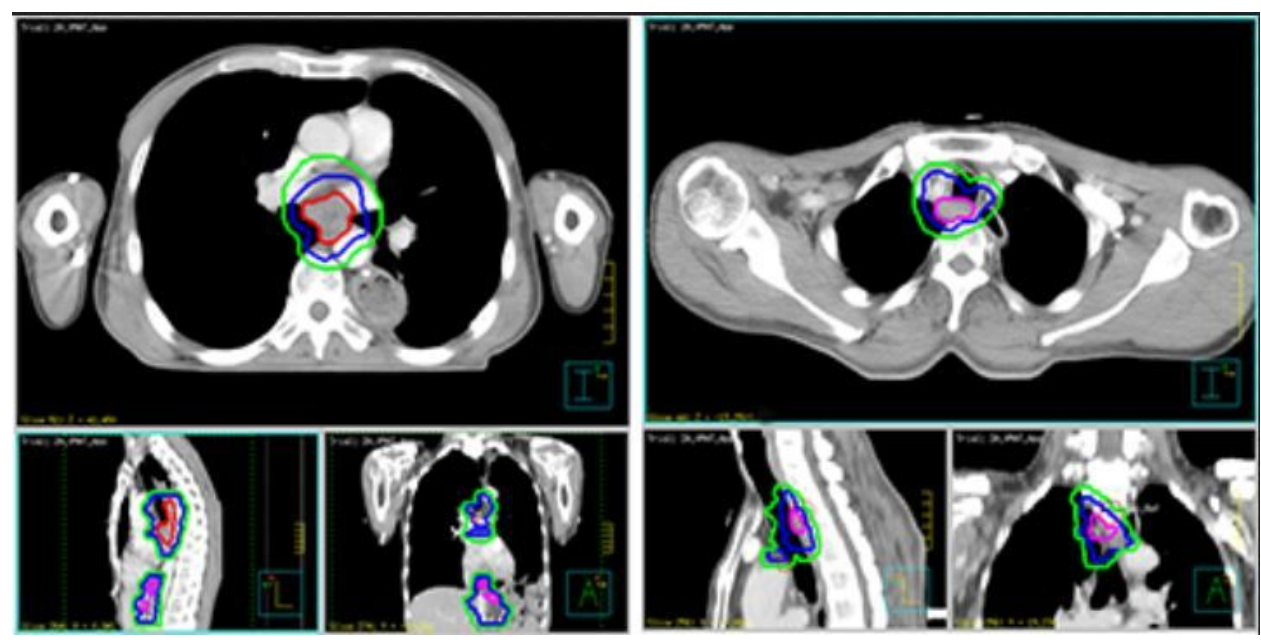

\section{Figure 4}

Examples of target volumes delineated in involved field irradiation (IFI) pattern are shown based on the planning computed tomography (CT) scans. (A) Lesions which were $>5 \mathrm{~cm}$ and far from the thoracic stomach recurred in Station 7, 8M/L and 15-17. The IFI field includes Station partial 4R/L, 7, 8U/M, and 15-17. (B) Lesions adjacent to the thoracic stomach recurred in Station $2 \mathrm{R}$ and palliative CRT was performed because of its time-to-recurrence $>16$ months. The IFI field only includes peritumor regions. Pink outlines GTV-N, Red outlines GTV-T, blue outlines CTV, and green outlines PTV. 